\title{
Research on Fire Detection method of Substation Based on Multi-sensors information Dynamic Tracing and Fusion Technology
}

\author{
Shu Ping Wang ${ }^{1,}$, , Hai Chao Wang ${ }^{2}$, Ming hao Fan ${ }^{1}$, Hai Cheng $\mathrm{Wu}^{1}$ and Jia \\ Qing Zhang ${ }^{1}$ \\ ${ }^{1}$ State Grid Anhui Electric Power Research Institute, No.73, Jinzhai Road, Hefei, Anhui Province, \\ 230022, China \\ ${ }^{2}$ State Grid Anhui Electric Power Corporation, No.9, Huangshan Road, Hefei, Anhui Province, \\ 230061, China \\ awangshuping516@126.com
}

Keywords: Substation, Multi-sensors, Fire detection, Information Dynamic Tracing, Information Fusion, BP neutral network

Abstract. The complex detectors with multi-sensors of feature parameters are developed for realizing early fire-detection in substation. The fire detecting signal measurements from these multi-sensors and their variation rates are used as the data of substation fire judgment. The time of fire alarm is advanced through tracing and calculating the variation rates of the detecting signals. The multi-detecting signals are processed and fused through Back Propagation (BP) neutral network, which not only avoids shortcomings of traditional fire detectors using a single fire signal as fire judgment but also reduces false positives and false negatives of fire. The training samples including training set and test set are built, and feedback correction for the samples ensures their rationality. In order to meet the stability of fire detection results in substation, batch mode for training is applied. Server push technology presented here accelerates speed of fire response further. Tests prove the feasibility and validity of the above mentioned ways, which means the accuracy and the speed of fire alarm in substation are greatly enhanced.

\section{Introduction}

At present most of fire detecting devices in substation are smoke and temperature detectors and this kind of detectors can detect only one parameter, such as smoke concentration or temperature [1]. Actually, in substation there are several combustibles including transformer oil, cables, electrical insulation paper and decorative materials etc.. Different feature parameters are produced during early stage of fire and different stages of fire development. Feature parameters include humidity, flame, concentrations of $\mathrm{CO}_{2}, \mathrm{SO}_{2}, \mathrm{HCl}$ and $\mathrm{NO}_{\mathrm{X}}$ besides temperature, $\mathrm{CO}$ and smoke concentration. Conventional detectors based on single parameter are influenced easily by environment leading to false negatives, and also can't detect these feature parameters leading to false positives [2].

For the above reasons, complex detectors with multi-sensors of feature parameters are developed for synchronous and comprehensive detecting multi-parameters of fire. Fusion technology of information for calculating and judging the multi-parameters and their variation rates are studied and dynamic tracing of fire is realized, so the efficiency and accuracy of fire alarm are enhanced. According to the test results, the method presented here is proved to be valid for increasing the ability of fire defense and safety in substation.

\section{Complex detectors}

Any two of the sensors of feature parameters are grouped into one complex detector as shown in Fig.1. The number and the types of the sensors depend on special size and combustibles. The bigger the special size is, the more sensors the number are needed. There are transformer oil, cables, insulation paper and decorative materials etc. Not only the temperature rises and the humidity changes, but also these combustibles produce $\mathrm{CO}, \mathrm{CO}_{2}, \mathrm{SO}_{2}, \mathrm{HCl}, \mathrm{NO}_{\mathrm{x}}$, flame, smoke when fire occurs in substation, 
so the types of the sensors are the ones of temperature, humidity, $\mathrm{CO}, \mathrm{CO}_{2}, \mathrm{SO}_{2}, \mathrm{HCl}, \mathrm{NO}_{\mathrm{X}}$, flame and smoke. Every detector mainly includes the modules of $\mathrm{A} / \mathrm{D}, \mathrm{CPU}$ and communication as shown in Fig.2. The characteristic parameters of fire gathered by the sensors are converted into digital signals through A/D module which can be received and processed by CPU. The processed signals of fire can be transmitted between the CPU and the computer through the communication and network. The information fusion technology is carried out by the computer.

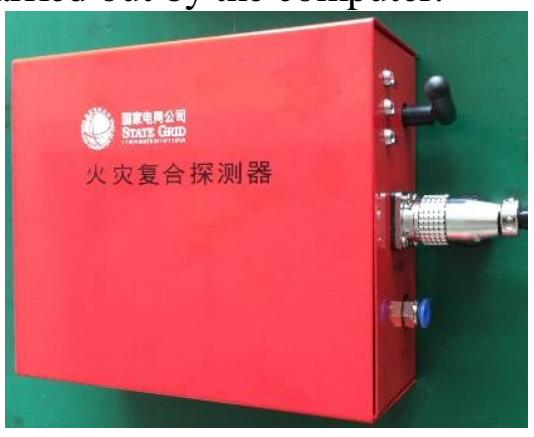

Fig.1 Outside view drawing of complex detector

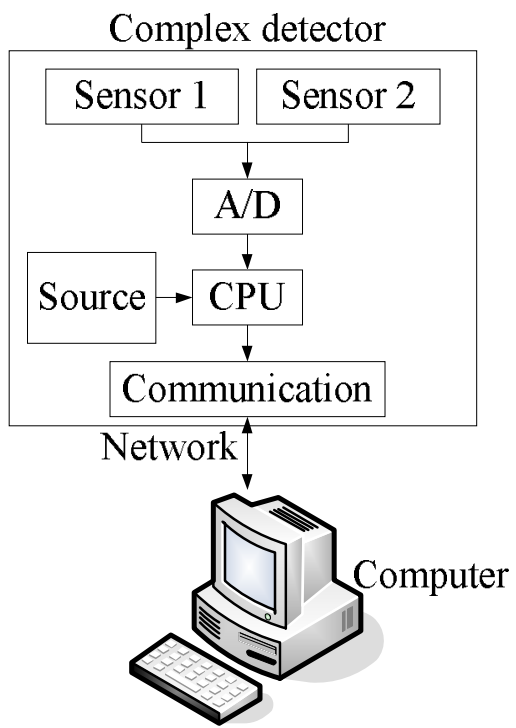

Fig.2 Functions of complex detector

\section{Information acquisition and pre-processing}

Usually the method of information fusion includes three layers of information, feature and decision [3]. As the basic layer of the three layers, Information layer is responsible for collecting fire signals and pre-processing these signals. Normally the signals for fire judgment should be temperature, humidity, flame, concentrations of $\mathrm{CO}, \mathrm{CO}_{2}, \mathrm{SO}_{2}, \mathrm{HCl}, \mathrm{NO}_{\mathrm{X}}$, and smoke, but this way easily leads to false positives and fire alarm delay. The detecting signals are influenced by environment. False positives occurs if the thresholds are set to be too low, because sometimes some environment values, like temperature and smoke concentration, also can reach the thresholds. Fire alarm delay occurs if the thresholds are set to be too high, because some values reach the thresholds after fire lasts a long time, which results in missing the best time to fight fire.

Actually, the measurements of fire characteristic parameters change constantly and the fluctuation of the measurements are great during fire. Temperature variation, for example, keeps low relief in normal condition, but it is great if fire occurs, so the collected data involves temperature, humidity, flame, concentrations of $\mathrm{CO}, \mathrm{CO}_{2}, \mathrm{SO}_{2}, \mathrm{HCl}, \mathrm{NO}_{\mathrm{X}}$, smoke and their variation rates except the flame. It means we can judge whether fire occurs or not when one of the variation rates is out of the limited 
value even though the signal value is low and thus false positives and delaying fire alarm delay can be avoided.

The data needs pre-processing after finishing data collecting, which means that checking whether the data is complete, there is bad data and there are logical contradictions among the data. The data will be abandoned if they are incomplete and need to be collected again. If there are partial absences or distortions existing in the data, they can be amended by interpolation to a group of complete data.

\section{Fusion of feature layer based on BP neutral network}

Modeling. Key problem is decision of inputs, outputs, hidden layer number, learning step, initial weights [4].

The inputs and outputs are in Table 1. Since the different ranges of the detectors cause a great deal of difference among these inputs, the inputs need to be normalized to the domain of [0,1], which avoids not only big data being drowned by small ones but also slowing BP correction due to the big difference among the inputs [4]. The normalized function is given as

$$
f(x)=\frac{1-e^{-\alpha\left(x-x_{0}\right)}}{1+e^{-\alpha\left(x-x_{0}\right)}}
$$

where $x_{0}$ is the minimum value of the range, $x$ is the value of fire parameter, $\alpha$ is the normalized coefficient.

Table 1 Inputs and outputs of BP neutral network

\begin{tabular}{|c|c|c|c|}
\hline \multicolumn{4}{|c|}{ Inputs } \\
\hline Parameters & Names & Parameters & Names \\
\hline$x_{1}$ & Temperature & $f x_{1}$ & Variation rate of temperature \\
\hline$x_{2}$ & Humidity & $f x_{2}$ & Variation rate of temperature \\
\hline$x_{3}$ & Flame & $f x_{3}$ & Variation rate of $\mathrm{CO}$ concentration \\
\hline$x_{4}$ & Concentration of $\mathrm{CO}$ & $f x_{4}$ & Variation rate of $\mathrm{CO}_{2}$ concentration \\
\hline$x_{5}$ & Concentration of $\mathrm{CO}_{2}$ & $f x_{5}$ & Variation rate of $\mathrm{HCl}$ concentration \\
\hline$x_{6}$ & Concentration of $\mathrm{HCl}$ & $f x_{6}$ & Variation rate of $\mathrm{SO}_{2}$ concentration \\
\hline$x_{7}$ & Concentration of $\mathrm{SO}_{2}$ & $f x_{7}$ & Variation rate of $\mathrm{NO}_{\mathrm{x}}$ concentration \\
\hline$x_{8}$ & Concentration of $\mathrm{NO}_{\mathrm{x}}$ & & \\
\hline \multicolumn{4}{|c|}{ Outputs } \\
\hline Parameters & Names & Parameters & Names \\
\hline$y_{1}$ & Lighted fire probability & $y_{4}$ & Dangerous probability of apparatus \\
\hline$y_{2}$ & Hidden fire probability & $y_{5}$ & Dangerous probability of persons \\
\hline$y_{3}$ & No fire probability & & \\
\hline
\end{tabular}

Here let $f(x)$ equal 0 when $x$ is the minimum value of the range and $f(x)$ equal 0.5 when $x$ reaches the alarm value, then $\alpha$ can be gotten. Considering personal safety, alarm values of every fire parameter are set as shown in Table 2. Taking temperature for example, its minimum value of the range and alarm value are $-20^{\circ} \mathrm{C}$ and $50^{\circ} \mathrm{C}$ respectively. Thus $\alpha$ is 0.0156945 , so the normalized function of temperature is expressed as

$$
f(x)=\frac{1-e^{-0.0156945(x+20)}}{1+e^{-0.0156945(x-20)}}
$$

The corresponding curve of formula (2) is shown in Fig.3. Supposing the range of temperature detector is $\left[-20^{\circ} \mathrm{C}, 1000^{\circ} \mathrm{C}\right]$ and temperature variation in substation is $\left[-20^{\circ} \mathrm{C}, 100^{\circ} \mathrm{C}\right]$, then the range of temperature variation only accounts for $11.8 \%$ of the total range, however, it accounts for $65.99 \%$ of the total range after being normalized seen from Fig.3. Thus the response sensitivity of temperature detector can be enhanced. Normalized way of other fire parameters is the same as that of temperature. We won't repeat again in this paper. 
Table 2 Alarm values of fire parameters

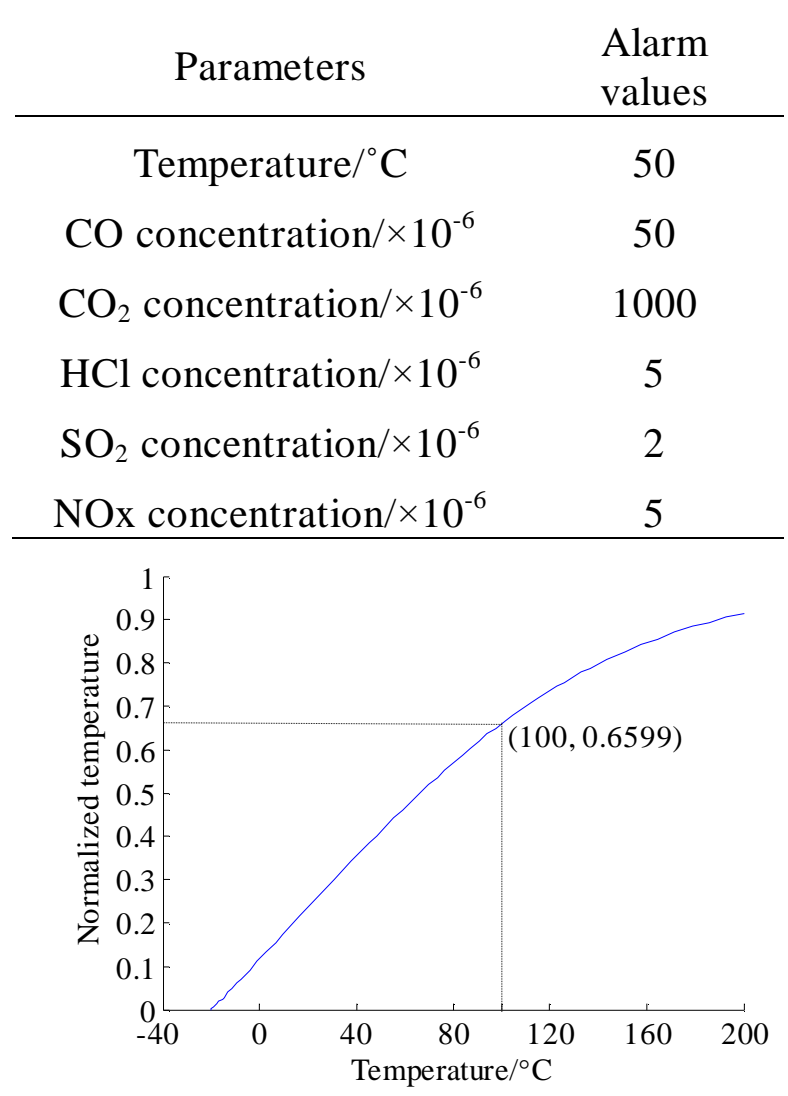

Fig.3 Normalized result of temperature

Suitable number of hidden layer is very important for BP's normal working. Too many or too few nodes of hidden layer are not helpful for BP training. According to experience and the method mentioned in the reference [5], the number of hidden layer is set to 12 here.

In learning algorithm, leaning step is between 0 and 1. Big learning step may accelerates convergence speed and upgrates weights faster, but sometimes it result in system oscilation. Small learning step can make the learning process stable but the learning speed slow [5]. In this system, initial learning step is set to 0.9 according to the experience and the actual test results, and then we can adjust dynamically according to the convergence.

Every weight and threshold initializations should be suitable when we start to learn, otherwise the learning accuracy and speed will be influenced. Initial weights can be random values between -1 and 1, and these values can't be equal at the same time [5].

Constructing of training sample. Fire has a great destructiveness, so it's unpractical to create training sample according the actual condition. In this system, the complete training sample is constructed through expert knowledge base which is based on not only the characteristic of the acquisition parameters in application scenario but also the acquisition information of historical fire accidents. The sample information of historical fire is divided into training set accounting for $67 \%$ of the total sample and test set accounting for $33 \%$ of the total sample. The sample data for training BP neutral network is generated by the training set. The test set is used for testing the network. The constructed training sample is reasonable if over the $90 \%$ test results coincide with the actual results, otherwise it's unreasonable and needs to be corrected.

In addition, environmental factors should be considered because some acquisition parameters such as temperature and humidity are easily affected by environment. The acquisition system can't discern the variation of these parameters is caused by the environment change or fire development. Bases of temperature and humidity are different in different seasons. Much error will be caused if the environment changing isn't considered. Therefore, temperature and humidity are corrected according to the variation of their averages at different time in order to accuracy of judgment. 
Training mode. There are two training modes for BP neutral work that are increasing and batch. Network weights and bias will be updated every time when the input data is submitted in increasing mode. Network weights and bias won't be updated until all the input data is submitted in batch mode. Detecting results of fire should be stable. Some extreme or abnormal training samples will cause large fluctuation of weights and bias. In this case, the stability of results will be destroyed if increasing mode is applied, but these problems can be avoided through batch mode. Therefore, batch mode is used for training so as to keep the results stable in fire detecting.

BP neutral network algorithm. BP neutral network algorithm consists of forward propagation of information and BP of error [6]. The procedure of BP neutral network in this system is shown in Fig.3. The process of learning and training won't be end until the error is minimum or meets the expectation through adjusting the weights and thresholds of every layer repeatedly. The system transfers from the state of learning to working after finishing adjusting the weights. The system receives the inputs coming from information layer and calculates the output information including the probabilities of no fire, lighted fire, hidden fire and the degree of fire hazard which are the input information of decision layer.

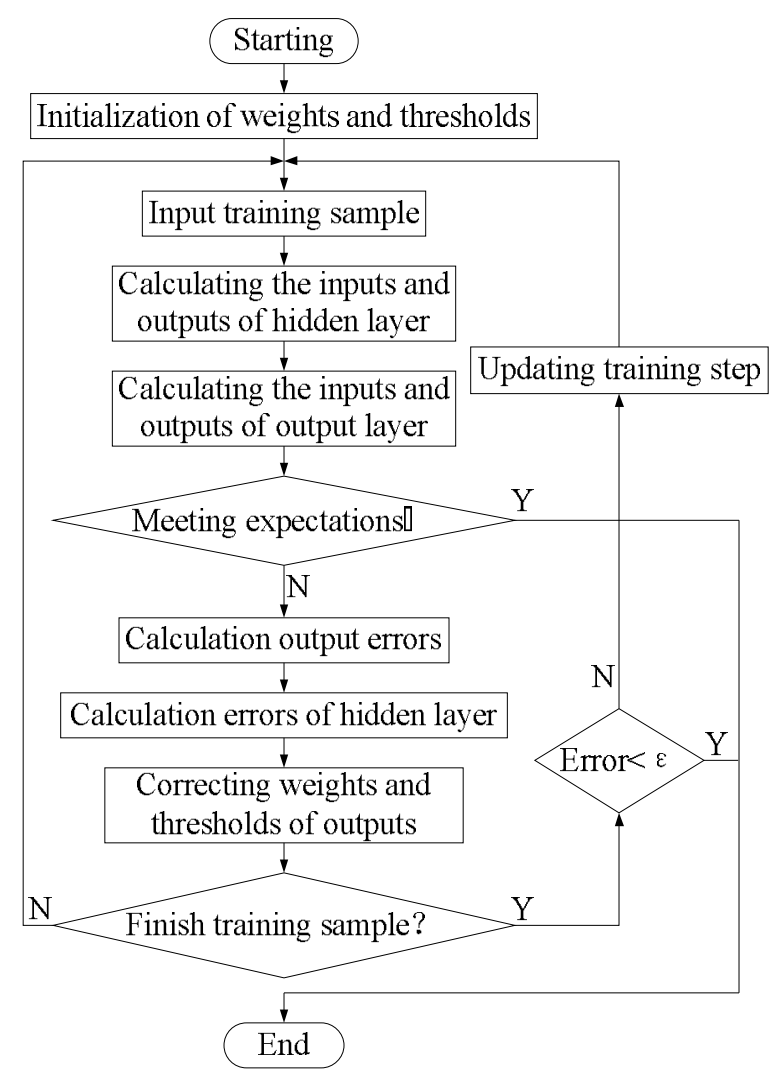

Fig.3 Flow chart of BP neutral network algorithm

Fuzzy logic inference. Fuzzy logic inference based on Mamdani is used for the information coming from feature layer and then the results of fuzzy logic inference are defuzzified through the center of gravity. Therefore, the accurate probabilities of no fire, lighted fire, hidden fire and the degree of fire hazard can be obtained.

\section{Test results and analysis}

Three groups of tests that simulate different combustible burning in substation have been done. The combustibles in test 1,2 and 3 are cables, decorative materials and firecrackers respectively. The measurements of every detector are shown in Fig.4. Among them, having flame or not is expressed with " 1 " and " 0 ". 

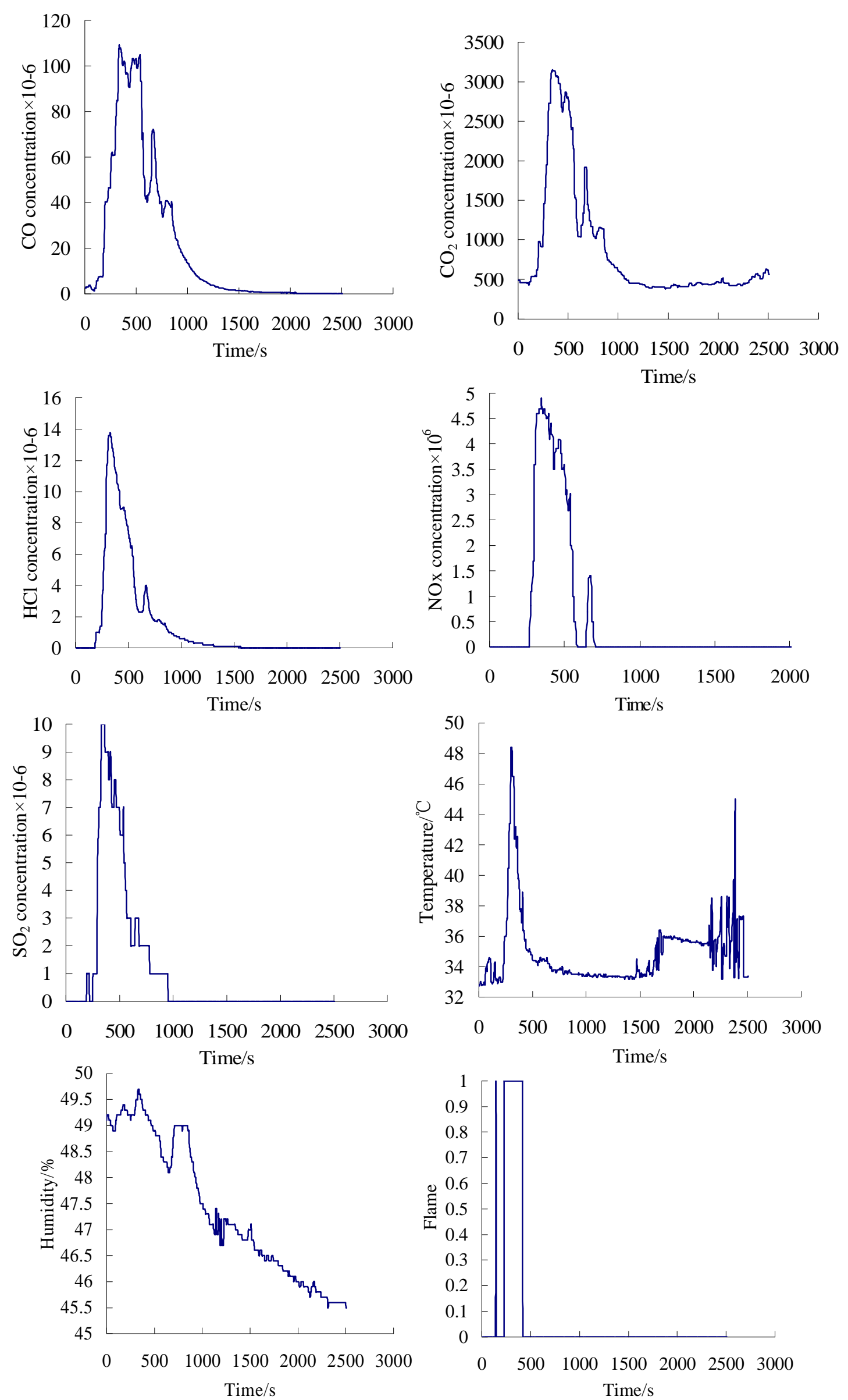

(a) Test 1 

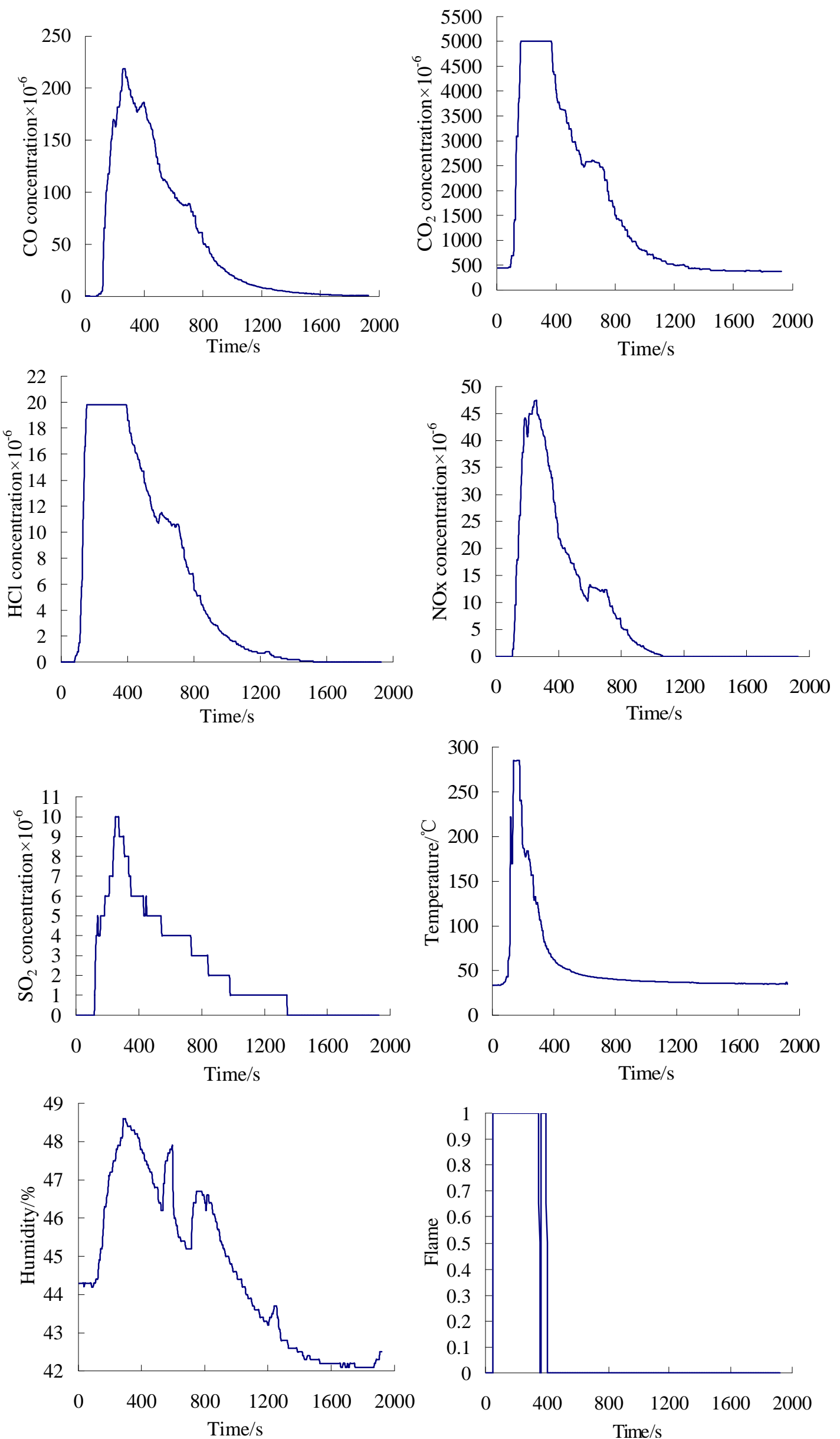

(b) Test 2 

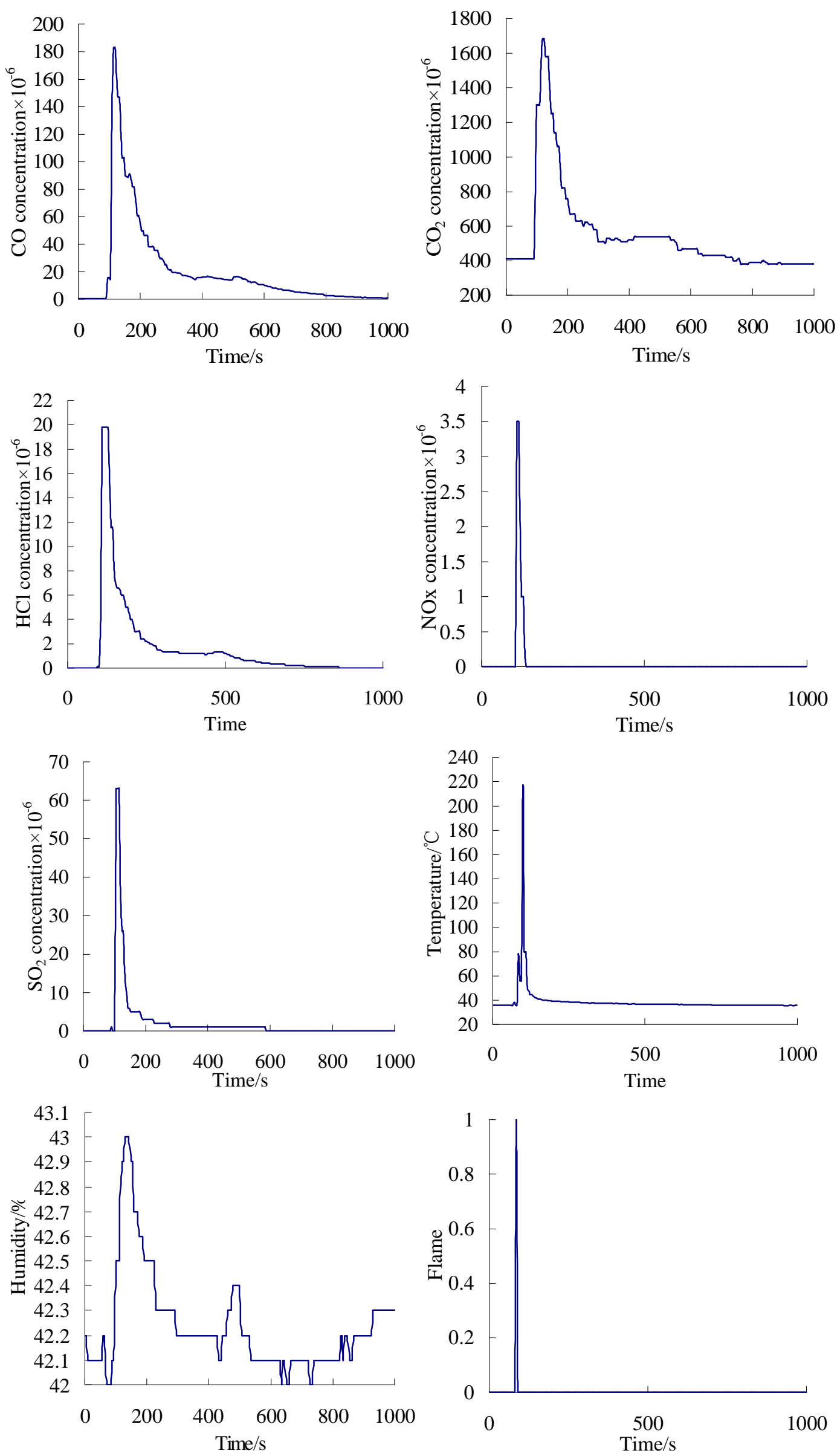

(c) Test 3

Fig.4 Variation of fire characteristic parameters 
Since different types of fire appear different characteristic, and the distance between detector and burning center influence the detecting result, false negative of fire will easily happen if using single information as the fire judgment. It can be seen in test 1 the temperature hasn't reached the alarm value $50^{\circ} \mathrm{C}$ although it rises obviously during the whole burning because the temperature detector is far away from the burning centre. If only temperature detectors were installed in the place of test 1 , other fire signals would be missed and there was no alarm signal. In test 3, the flame and NOx detectors have not given out alarm although lighted firecrackers release NOx because the NOx concentration hasn't reached the alarm value $5 \times 10^{-6}$ and hidden fire has appeared. If only flame and NOx detectors were installed in the place of test 3 , other fire signals would be missed which causes false negative. Therefore, multi-detectors are applied in the system for avoiding false negative.

Alarm time of every detector in the three groups of tests is shown in Table 3. In test 1, the flame detector alarms first and alarm happens one time at $144 \mathrm{~s}$. Then alarm stops until it starts alarming continuously at $229 \mathrm{~s}$. However, alarm happens at $116 \mathrm{~s}$, which alarms 28 s earlier than usual through fusion of dynamic information. The alarm time of the flame detector after using fusion technology of dynamic information happens at 52s which is the same as that of traditional detecting method in test 2. In test 3, the temperature detector alarms first and alarm happens at 84s. However, after using fusion technology of dynamic information as the fire judgment, the alarm time is $11 \mathrm{~s}$ in advance. According to the results, it can be seen the alarm time is in advance obviously after dynamic tracing and fusion technology of information are applied for the fire judgment, which is very significant for fighting fire in substation timely.

Table3 Fire alarm time of three groups of tests/s

\begin{tabular}{|c|c|c|c|c|c|c|c|c|c|}
\hline & \multicolumn{8}{|c|}{ Traditionl detecting method } & \multirow{2}{*}{$\begin{array}{c}\text { Dynamic tracing } \\
\text { and fusion } \\
\text { technology of } \\
\text { information } \\
\begin{array}{c}\text { Earliest alarm } \\
\text { time }\end{array}\end{array}$} \\
\hline & $\mathrm{CO}$ & $\mathrm{CO} 2$ & $\mathrm{HCl}$ & NOx & $\mathrm{SO}_{2}$ & Temperature & Humidity & Flame & \\
\hline Test 1 & 250 & 251 & 265 & - & 293 & - & - & $\begin{array}{c}\text { 144(Alarm first) } \\
\text { 229(Alarm } \\
\text { continously) }\end{array}$ & 116 \\
\hline Test 2 & 126 & 116 & 121 & 118 & 122 & 101 & - & 52 & 52 \\
\hline Test 3 & 107 & 98 & 109 & - & 107 & 84 & - & 86 & 73 \\
\hline
\end{tabular}

\section{Conclusions}

In this paper, fire detecting based on multi-parameters from complex detectors in substation was realized through adopting BP neutral network, which avoids many disadvantages brought by single sensor detecting and enhances the fire alarm accuracy. Trace of dynamic fire information advances the fire alarm time markedly, which is very important for detecting early fire and reduces fire breaking out. Three groups of tests through burning different combustibles have proved the availability and validity of the method studied in the paper.

\section{References}

[1] M.J.A. Khan, M.R. Imam and M.A.R. Sarkar: Automated Fire Fighting System with Smoke and Temperature detection (2012 $7^{\text {th }}$ International Conference on ICECE)

[2] D.L. Hall and J Llinas: Proceedings of the IEEE Vol. 85(1) (1999), p. 6 
[3] E. Zervas, A. Mpimpoudis, C. Anagnostopoulos, O. Sekkas and S. Hadjiefthymiads: Information Fusion Vol. 12(3) (2011), p. 150

[4] S. Chen, H. Bao, X. Zeng and Y. Yang: A Fire detecting method based on multi-sensor data fusion (2003 IEEE International Conference on System, Man \& Cybernetics)

[5] L.P. Wang: Research on Fire Detection Systme Based on Multi-sensors Information Fusion Technology. Hunan University, 2006 (in Chinese)

[6] T. Fujinaka, M. Yoshioka, S. Omatu and T. Kosaka: Intelligent Electronic Nose System for Fire Detection Systems Based on Neutral Networks (2008 $2^{\text {th }}$ International Conference on ADVCOMP) 